\title{
Interventional multi-spectral photoacoustic imaging in laparoscopic surgery
}

\author{
Emma R. Hill ${ }^{1}$, Wenfeng Xia ${ }^{1}$, Daniil I. Nikitichev ${ }^{1}$, Kurinchi Gurusamy ${ }^{2}$, Paul C. Beard ${ }^{1}$, \\ David J. Hawkes ${ }^{1}$, Brian R. Davidson ${ }^{2}$ and Adrien E. Desjardins ${ }^{1}$ \\ ${ }^{1}$ Department of Medical Physics and Biomedical Engineering, University College London, \\ Gower street, London WC1E 6BT, United Kingdom; \\ ${ }^{2}$ HPB Surgery, Royal Free Hospital, Pond St, London NW3 2QG, United Kingdom;
}

\begin{abstract}
Laparoscopic procedures can be an attractive treatment option for liver resection, with a shortened hospital stay and reduced morbidity compared to open surgery. One of the central challenges of this technique is visualisation of concealed structures within the liver, particularly the vasculature and tumourous tissue. As photoacoustic (PA) imaging can provide contrast for haemoglobin in real time, it may be well suited to guiding laparoscopic procedures in order to avoid inadvertent trauma to vascular structures. In this study, a clinical laparoscopic ultrasound probe was used to receive ultrasound for PA imaging and to obtain co-registered B-mode ultrasound (US) images. Pulsed excitation light was delivered to the tissue via a fibre bundle in dark-field mode. Monte Carlo simulations were performed to optimise the light delivery geometry for imaging targets at depths of 1 $\mathrm{cm}, 2 \mathrm{~cm}$ and $3 \mathrm{~cm}$, and 3D-printed mounts were used to position the fibre bundle relative to the transducer according to the simulation results. The performance of the photoacoustic laparoscope system was evaluated with phantoms and tissue models. The clinical potential of hybrid PA/US imaging to improve the guidance of laparoscopic surgery is discussed.
\end{abstract}

Keywords: photoacoustic imaging; ultrasound imaging; laparoscopy; image guidance; liver; minimally invasive procedures

\section{INTRODUCTION}

Many abdominal procedures make use of laparoscopic surgery, in which the surgeon operates through small incisions (5 - $12 \mathrm{~mm}$ diameter) using specialised tools. This approach leads to a shortened hospital stay, reduced pain and decreased morbidity. In spite of these advantages, laparoscopy has not been widely taken up in liver resection. This is primarily because the liver is highly vascular, with blood entering the liver from two major vascular systems: the hepatic artery and portal vein. In laparoscopy, it is difficult to visualise sub-surface structures using a standard videoscope, making it more likely that the surgeon will cut through a major vessel, which may be harder to control when surgery is carried out through a small access port. ${ }^{1}$

Identification of sub-surface features can be performed using ultrasound. Clinical ultrasound transducers that are suitable for laparoscopic imaging are already being manufactured and used in surgery. However, acoustic contrast is limited and it can be difficult to distinguish between tissue structures such as blood vessels, nerves and the bile duct. Optical techniques can give greater contrast due to the characteristic scattering and absorption properties of soft tissue targets, but have limited imaging depth as light is strongly scattered in tissue.

Photoacoustic (PA) imaging is able to couple the good tissue contrast of optical modalities with the spatial resolution of US imaging. Tissue is irradiated with pulsed light, which is scattered throughout the tissue and absorbed by chromophores. This is followed by a rapid increase in local temperature which leads to US generation. ${ }^{2}$ The generated ultrasound waves can be detected using the same ultrasound transducer that is used to obtain US images, so the two imaging modalities can be acquired in an inherently co-registered manner. A hybrid PA/US system has the potential to provide both structural information from US as well as molecular information from PA.

Address all correspondence to: Emma Hill, emma.hill.14@ucl.ac.uk.

Photons Plus Ultrasound: Imaging and Sensing 2016, edited by Alexander A. Oraevsky, Lihong V. Wang Proc. of SPIE Vol. 9708, 97080B - (C) 2016 SPIE · CCC code: 1605-7422/16/\$18 · doi: 10.1117/12.2208428 
There has been growing interest in developing photoacoustic imaging systems for guidance of surgical procedures. $^{3-7}$ Xia et al. ${ }^{8}$ reported a PA imaging system for needle guidance in which excitation light was delivered directly through an optical fibre embedded in a needle. Kim et al. ${ }^{4}$ reported a PA imaging system based on a commerical US system for guidance of sentinel lymph node biopsies, in which excitation light was delivered via a bifurcated fibre bundle integrated into the ultrasound transducer housing.

We propose a new application for hybrid PA/US imaging in guidance of laparoscopic procedures, including liver resection. Towards this goal, a system was developed in which US detection was performed with a commercial laparoscopic imaging probe. Excitation light was delivered via a fibre bundle which can be miniaturised for clinical application. Co-registered photoacoustic and ultrasound images were obtained to provide complementary images of tissue. The system was tested using phantoms, an in vivo vessel model, and ex vivo porcine liver.

There have been some previous attempts to obtain PA images of the liver. Miyata et al. ${ }^{9}$ used ICG to gain contrast for tumourous tissue in the liver, while An et al. ${ }^{10}$ used squaraine dye for this application. While these results are promising, imaging the vasculature in the liver remains a challenge because the liver tissue itself has a similar absorption spectrum to deoxygenated haemoglobin, ${ }^{11}$ making it difficult to obtain contrast between the vessels and the surrounding tissue.

In this study, two mechanisms for obtaining contrast were considered. In the first, a single excitation wavelength in the near-infrared range was used $(1064 \mathrm{~nm})$. This mechanism has a greater imaging depth as light is less strongly absorbed in this range. However, there is limited specificity in differentiation of vessels and other structures. The second mechanism for obtaining contrast is to use multispectral imaging, acquiring across the 750 - $850 \mathrm{~nm}$ wavelength range to improve the contrast between oxygenated and deoxygenated blood. While this has greater specificity for tissue targets, care is needed for accurate interpretation of results, given the wavelength-dependence of light propagation in tissue.

\section{MATERIALS AND METHODS}

\subsection{The imaging system}

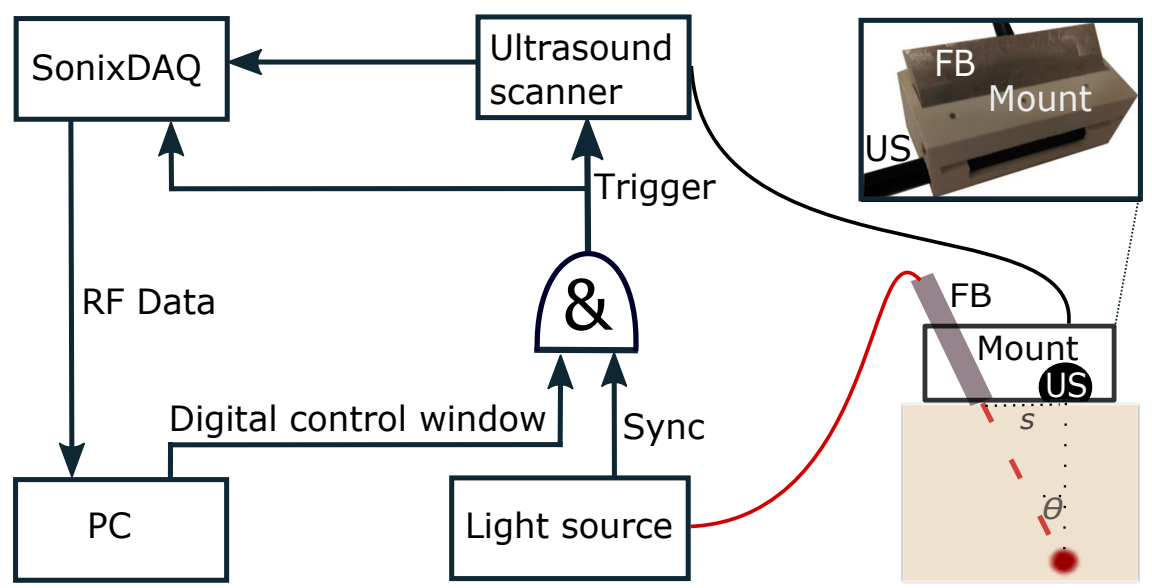

Figure 1: The PA/US hybrid imaging system. Excitation light is delivered via an angled fibre bundle (labelled FB) to the tissue surface. PA and US data are acquired by a clinical laparoscopic ultrasound transducer (labelled US) and reconstructed to produce co-registered images. The fibre bundle and transducer are held in a fixed geometry by a 3D printed mount, as pictured in the inset image. The separation of the probe and fibre bundle is denoted by $s$, and the incident angle of the excitation light is represented as $\theta$.

A prototype system was developed based on a clinical laparoscopic ultrasound scanner, as shown in Figure 1. Excitation light was delivered in dark-field mode via an angled fibre bundle that converted the incident light from 
a single multi-mode fibre into a uniform rectangular beam pattern at the tissue surface. Dark-field illumination was used here as it reduces interference caused by strong PA signal from superficial features.

Monte Carlo simulations were performed using the mcxyz implementation ${ }^{12}$ for a $3 \mathrm{D}$ model. The optical properties were chosen to match the optical properties of $1 \%$ Intralipid $^{13,14}\left(1000 \mathrm{~nm}, \mu_{a}=2 \mathrm{~mm}^{-1}, \mu_{s}=2\right.$ $\left.\mathrm{mm}^{-1}, \mathrm{~g}=0.5\right)$. Three light delivery geometries were selected for imaging targets at depths of $1 \mathrm{~cm}, 2 \mathrm{~cm}$ and $3 \mathrm{~cm}$. 3D printed holders were built to hold the laparoscopic probe and the fibre bundle in the fixed geometries, as pictured in Figure 1.

Two lasers were used as excitation light sources. An Nd:YAG laser $(1064 \mathrm{~nm}$, pulse width $8 \mathrm{~ns}$, repetition rate $20 \mathrm{~Hz}$, Ultra 50, Quantel) was used for single wavelength imaging in the near-infrared range. For multispectral imaging, an optical parametric oscillator (OPO) system (VersaScan L-532, GWU-Lasertechnik) was pumped by a frequency-doubled Q-switched Nd:YAG laser (pulse width $6 \mathrm{~ns}$, repetition rate $10 \mathrm{~Hz}$, Quanta-Ray, INDI-40-10, Spectra-Physics), imaging in the 750 - $850 \mathrm{~nm}$ range. The outputs were coupled with silica-silica optical fibres (core diameter $910 \mu \mathrm{m}$ ) to the fibre bundle. The pulse energy was measured by a photodetector, which received $4 \%$ of the laser output. The pulse energy of the multispectral system did not exceed $5 \mathrm{~mJ}$ while the pulse energy of the single wavelength system did not exceed $50 \mathrm{~mJ}$.

US detection was performed using a commercial US imaging system (SonixMDP, Analogic Ultrasound) operated in its research mode. Here, a laparoscopic linear array imaging probe (Lap-9-4/38, 4 - $9 \mathrm{MHz}$, Vermon) was used to obtain the ultrasound data. Pre-beamformed channel data from the transducer was sampled by a 128-channel data acquisition system (SonixDAQ, Analogic Ultrasound) at a sample frequency of $80 \mathrm{MHz}$ and the data transferred to a PC. PA and US acquisition was interleaved and triggered optically using the gating system outlined by Xia et al. ${ }^{8}$

Reconstruction was performed using delay-and-sum beamforming. Further PA image reconstruction was performed using a Fourier Transform method, implemented offline using the k-Wave MATLAB toolbox. ${ }^{15}$

\subsection{Imaging targets}

The relationship between the PA signal amplitude and target depth was determined using a vessel phantom consisting of a tube filled with India ink $\left(0.5 \%, \mu_{a}=0.45 \mathrm{~mm}^{-1}\right)$, immersed in a $1 \%$ Intralipid solution to simulate the optical scattering properties of tissue. The position of the tube was fixed, and the hybrid imaging probe translated in the vertical direction to vary the target depth from $10 \mathrm{~mm}$ to $35 \mathrm{~mm}$. PA images were obtained at $1064 \mathrm{~nm}$ for each depth and the mean signal in the ROI corresponding to the tube calculated.

The system was further tested using the vessels in the forearm as an in vivo vascular model. Multi-spectral images were obtained using five excitation wavelengths between $750 \mathrm{~nm}$ and $850 \mathrm{~nm}$ (25 nm steps).

Finally, ex vivo porcine liver tissue was used as a tissue target, with image acquisition performed immediately post mortem. A gel wax pad (1 cm thickness) was used to separate the hybrid imaging probe from the tissue surface so that artefacts due to reflections at the transducer interface would be separable from the signal produced at the tissue surface. Imaging was performed with a single excitation wavelength of $1064 \mathrm{~nm}$.

\section{RESULTS AND DISCUSSION}

As light delivery in tissue is not completely diffuse, there was a need to optimise the light delivery geometry to maximise fluence at the target. Through Monte Carlo simulations, it was found that the optimal incident angle of the excitation light depends on the imaging depth. As shown in Figure 2, for superficial targets (depth < $1.2 \mathrm{~cm}$ ), the fluence is higher for large incident angles, while smaller angles are preferable when deep imaging is required. The incident angle of the excitation light and the separation of the probe and fibre bundle chosen for the three imaging depths are given in Table 1. 3D printed holders were built to fix the fibre bundle and probe in these geometries.

The three imaging geometries of the laparoscopic system were tested using a vessel phantom, which consisted of a tube filled with India ink immersed in Intralipid. It should be noted that while Intralipid is a good model for scattering, it is less absorbing than real tissue. Example co-registered PA and US images of the phantom are given in Figure 3. The PA signal is strongest in the upper and lower boundaries of the tube, and there is a 
(a)

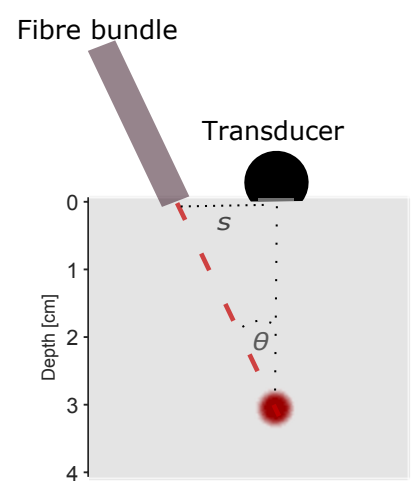

(b)

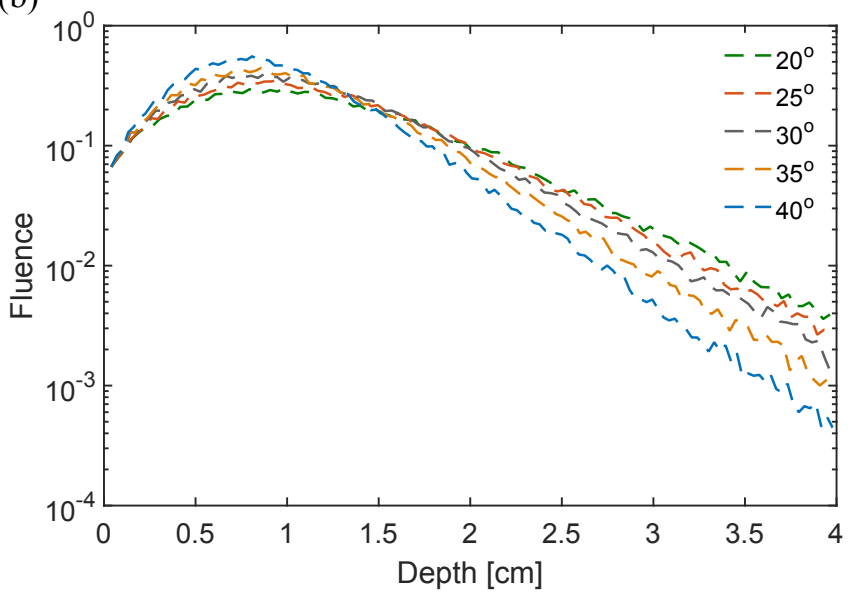

Figure 2: Monte Carlo simulations were performed using a 3D tissue model with varying incident angle $\theta$ and separation of the fibre bundle and probe $s$. (b) shows how the fluence profiles vary for different incident angles of the excitation light when $s=10 \mathrm{~mm}$. Three geometries were selected according to the simulation results and 3D printed mounts were built, as detailed in Table 1.

Table 1: Three geometries were selected according to the simulation results for imaging targets at depths of $1 \mathrm{~cm}, 2 \mathrm{~cm}$ and $3 \mathrm{~cm}$. The separation of the fibre bundle and transducer, $s$, was chosen to be $10 \mathrm{~mm}$ in each case.

\begin{tabular}{lll} 
Holder & Angle $\left.{ }^{\circ}{ }^{\circ}\right]$ & Target imaging depth $[\mathrm{cm}]$ \\
\hline \hline 1 & 40 & 1.0 \\
2 & 25 & 2.0 \\
3 & 20 & 3.0
\end{tabular}

(a)

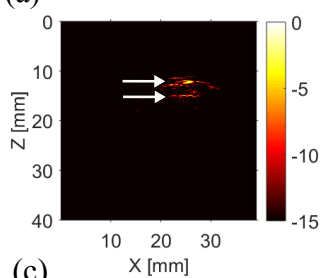

(c)

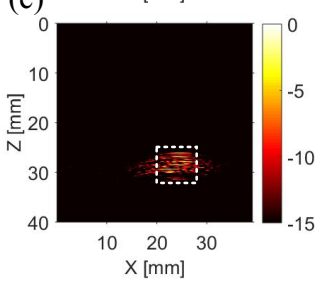

(b)

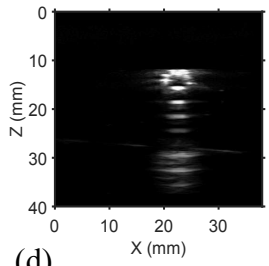

(d)

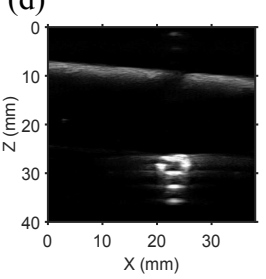

(e)

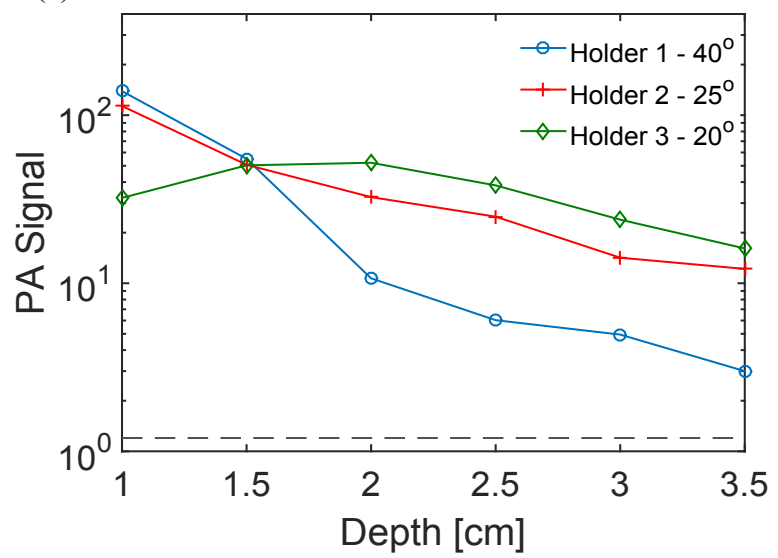

Figure 3: A vessel phantom consisted of a tube filled with India ink (0.5\%), immersed in Intralipid $(1 \%)$. The height was varied and images were obtained at each depth for the three excitation geometries, corresponding to three probe holders (as detailed in $\mathrm{T}$ able 1). Representative PA and US images are given in $(\mathrm{a}, \mathrm{c})$ and $(\mathrm{b}, \mathrm{d})$, respectively. The PA signal is strongest in the upper and lower tube boundaries, as indicated by the arrows in (a). The cross-sectional area of the tube was treated as a region of interest (ROI), as indicated by the dashed boundary in (c) and the signal averaged across the ROI for the three excitation geometries. The results, along with the noise floor (dashed), are given in (e). 
good degree of spatial correspondence between the PA and US images. The signals corresponding to the three imaging geometries are plotted as a function of depth in Figure 3. As predicted in simulation, the largest angle (Holder 1) is best for superficial imaging, while the smallest angle (Holder 3) gives the greatest PA signal when imaging deeper targets. Our results indicate that for clinical applications, the geometry of the imaging probe should be chosen according to the required imaging depth.
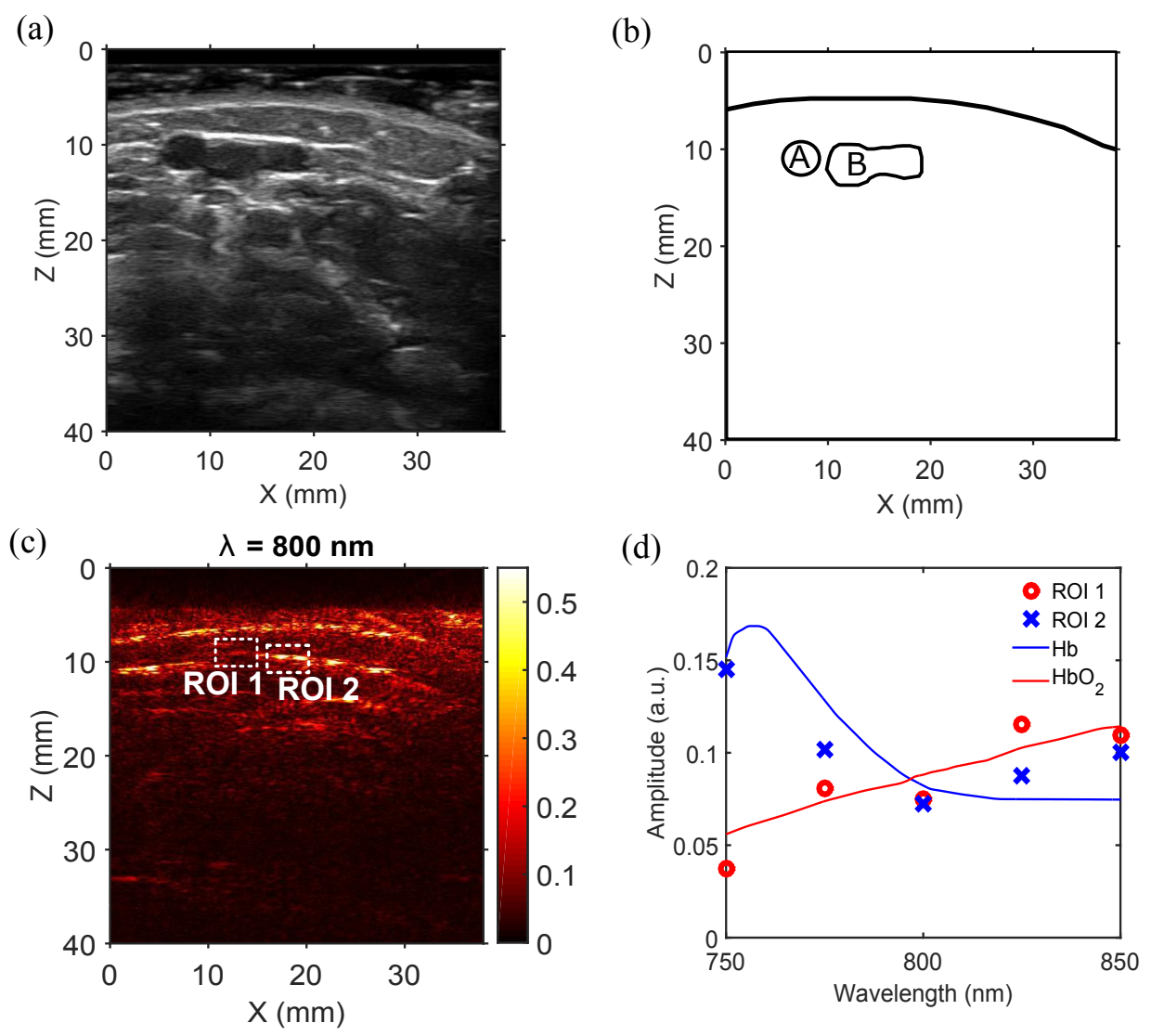

Figure 4: The multispectral capabilities of the system were tested using an in vivo forearm as a vascular model. Excitation light was provided at five wavelengths between 750 and $850 \mathrm{~nm}$. The ultrasound image is annotated in (b) to show the brachial artery (A) and the basilic vein (B). The reconstructed PA image for $800 \mathrm{~nm}$ is shown in (c). The PA signal was averaged in the two highlighted regions of interest (ROI 1 and ROI 2) and is plotted as a function of wavelength in (d). The absorption spectrum of oxygenated and deoxygenated haemoglobin is given in (d) for comparison, ${ }^{16}$ normalised to the mean magnitude of the measured signal in ROI 1.

The hybrid imaging system was used to image the vessels in the forearm as an in vivo vascular model for multispectral imaging. Example US and PA images are given in Figure 4, and the PA signal in two regions of interest is plotted as a function of wavelength in Figure 4(c). Even with a small number of acquisition wavelengths, the spectra appear to correspond well to the absorption spectra of oxygenated and deoxygenated haemoglobin. This is promising and suggests that PA/US imaging has the potential to obtain contrast for blood oxygenation when a suitable inversion scheme is used.

Finally, the system was used to image ex vivo porcine liver tissue at a wavelength of $1064 \mathrm{~nm}$, with representative PA and US images given in Figure 5. 


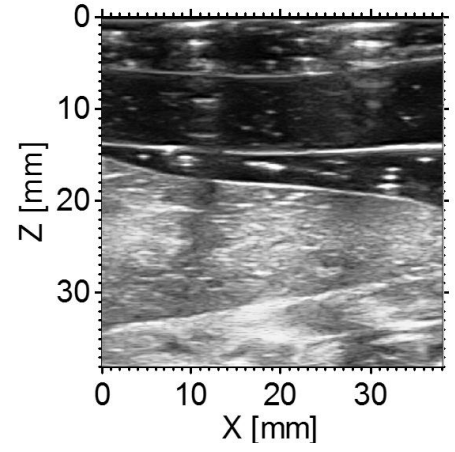

(a)

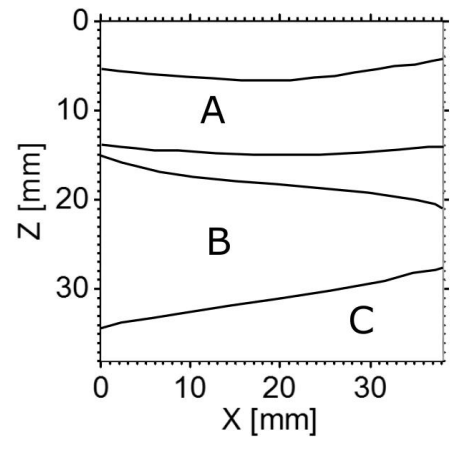

(b)

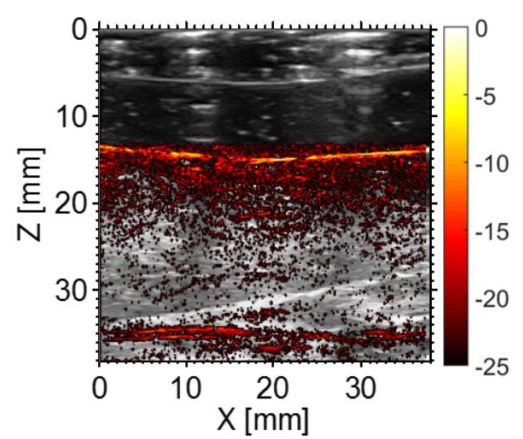

(c)

Figure 5: PA/US imaging $(1064 \mathrm{~nm})$ of a swine liver, imaged immediately post mortem. The ultrasound is given in (a) and annotated in (b). (A) indicates a gel wax pad with ultrasound gel either side, used to separate the probe from the tissue surface, while $(\mathrm{B})$ and $(\mathrm{C})$ are two lobes of the liver

\section{CONCLUSIONS}

A photoacoustic imaging system based on a clinical laparoscopic ultrasound probe was introduced. Excitation light was delivered in the dark-field mode via a fibre bundle which can be miniaturised for clinical applications. Structural features were clearly visible at depths exceeding $20 \mathrm{~mm}$ in both the in vivo forearm model and the ex vivo liver tissue with $1064 \mathrm{~nm}$ excitation. Multispectral imaging results with the in vivo vascular model were promising and suggest that the laparoscopic system may be able to provide contrast for blood oxygenation in clinical applications. These results indicate that PA imaging could result in safer laparoscopic procedures, with reduced risk of vascular injury. With further optimisation and development, PA/US hybrid imaging has the potential to improve guidance of laparoscopic surgery.

\section{ACKNOWLEDGMENTS}

This work was supported by the EPSRC-funded UCL Centre for Doctoral Training in Medical Imaging, the Department of Health's NIHR-funded Biomedical Research Centre at University College London Hospitals and by a Starting Grant from the European Research Council [ERC-2012-StG, Proposal 310970 MOPHIM].

\section{REFERENCES}

[1] Tranchart, H., O'Rourke, N., Van Dam, R., Gaillard, M., Lainas, P., Sugioka, A., Wakabayashi, G., and Dagher, I., "Bleeding control during laparoscopic liver resection: a review of the literature," Journ. HPB Sci. 22(5), 371-378 (2015).

[2] Beard, P., "Biomedical photocoustic imaging," Interface Focus 1(4), 602-631 (2011).

[3] Song, K., Stein, E., Margenthaler, J., and Wang, L., "Noninvasive photoacoustic identification of sentinel lymph nodes containing methylene blue in vivo in a rat model," J. Biomed. Opt. 13(5), 054033 (2008).

[4] Kim, C., Erpelding, T., Maslov, K., Jankovic, L., Akers, W., Song, L., Achilefu, S., Margenthaler, J., Pashley, M., and Wang, L., "Handheld array-based photoacoustic probe for guiding needle biopsy of sentinel lymph nodes," J. Biomed. Opt. 15(4), 046010 (2010).

[5] Lediju Bel, M., Guo, N., Song, D., Kang, J., and Boctor, E., "in vivo visualization of prostate brachytherapy seeds with photoacoustic imaging," J. Biomed. Opt. 19(12), 126011 (2014).

[6] Lin, L., Xia, J., Wong, T., Li, L., and Wang, L., "in vivo deep brain imaging of rats using oral-cavity illuminated photoacoustic computed tomography," J. Biomed. Opt. 20(1), 016019 (2015). 
[7] Garcia-Uribe, A., Erpelding, T., Krumholz, A., Ke, H., Maslov, K., Appleton, C., Margenthaler, J., and Wang, L., "Dual-modality photoacoustic and ultrasound imaging system for noninvasive sentinel lymph node detection in patients with breast cancer," Scientific Reports 5, 15748 (2015).

[8] Xia, W., Nikitichev, D., Mari, J., West, S., Pratt, R., David, A., Ourselin, S., Beard, P., and Desjardins, A., "Performance characteristics of an interventional multispectral photoacoustic imaging system for guiding minimally invasive procedures," J. Biomed. Opt. 20(8), 086005 (2015).

[9] Miyata, A., Ishiwaza, T., Kamiya, M., Shimuzu, A., Kaneko, J., Ijichi, H., Shibahara, J., Fukayama, M., Midorikawa, Y., Urano, Y., and Kokudo, N., "Photoacoustic tomography of human hepatic malignancies using intraoperative indocyanine green fluorescence imaging.," PLoS One 9(11), 112667 (2014).

[10] An, F., Deng, Z., Zhang, J., Yang, Y., Li, C., Zheng, C. and Zhang, X., "Aggregation-induced near-infrared absorption of squaraine dye in an albumin nanocomplex for photoacoustic tomography in vivo.," ACS Appl. Mater. Interfaces 6(20), 17985-92 (2014).

[11] Ritz, J., Roggan, A., Isbert, C., Muller, G., Buhr, H., and Germer, C., "Optical properties of native and coagulated porcine liver tissue between 400 and 2400 nm.," Lasers Surg. Med. 29(3), 205-12 (2001).

[12] Jacques, S. and Li, T. "Monte Carlo simulations of light transport in 3D heterogeneous tissues (mcxyz.c)," http://omlc.org/software/mc/mcxyz/index.html (2013).

[13] Di Ninni, P., Martelli, F., and Zaccanti, G., "Effect of dependent scattering on the optical properties of Intralipid tissue phantoms," Biomed. Opt. Express 2(8), 2265-2278 (2011).

[14] van Staveren, H., Moes, C., van Marie, J., Prahil, S., and van Gemert, M., "Light scattering in Intralipid $10 \%$ in the wavelength range of $400-1100 \mathrm{~nm}, "$ Applied Optics 30(31), 4507-4514 (1991).

[15] Treeby, B. and Cox, B., "k-Wave: MATLAB toolbox for the simulation and reconstruction of photoacoustic wave fields," J. Biomed. Opt. 15(2), 021314 (2010).

[16] Nachabe, R., Hendriks, B., Desjardins, A., van der Voort, M., van der Mark, M., Sterenborg, H., "Estimation of lipid and water concentrations in scattering media with diffuse optical spectroscopy from 900 to $1600 \mathrm{~nm}$.," J. Biomed. Opt. 15(3), 037015 (2010). 